\title{
Desensitization of ABA-Signaling: The Swing From Activation to Degradation
}

\author{
Akhtar Ali ${ }^{1}$, Jose M. Pardo ${ }^{2}$ and Dae-Jin Yun ${ }^{1 *}$ \\ ${ }^{1}$ Department of Biomedical Science and Engineering, Konkuk University, Seoul, South Korea, ${ }^{2}$ Instituto de Bioquímica \\ Vegetal y Fotosíntesis, cicCartuja, CSIC-Universidad de Sevilla, Seville, Spain
}

Abscisic acid (ABA) is a key plant stress-signaling hormone that accumulates upon osmotic stresses such as drought and high salinity. Several proteins have been identified that constitute the ABA-signaling pathway. Among them ABA receptors (PYR/PYL/RCAR), co-receptor PP2Cs (protein phosphatases), SnRK2 kinases (SNF1-related protein kinases) and ABI5/ABFs (transcription factors) are the major components. Upon ABA signal, PYR/PYL receptors interact with and recruit PP2Cs, releasing SnRK2s kinases from sequestration with PP2Cs. This allows SnKR2s to promote the activation of downstream transcription factors of ABA pathway. However,

OPEN ACCESS

Edited by:

Taishi Umezawa,

Tokyo University of Agriculture and Technology, Japan

Reviewed by:

Jigang Li,

China Agricultural University, China Jun Yan,

East China Normal University, China

*Correspondence:

Dae-Jin Yun

djyun@konkuk.ac.kr

Specialty section:

This article was submitted to

Plant Abiotic Stress,

a section of the journal

Frontiers in Plant Science

Received: 11 November 2019

Accepted: 17 March 2020

Published: 22 April 2020

Citation:

Ali A, Pardo JM and Yun D-J (2020) Desensitization

of ABA-Signaling: The Swing From

Activation to Degradation.

Front. Plant Sci. 11:379.

doi: 10.3389/fpls.2020.00379 apart from activation, ubiquitination and degradation of core proteins in the ABA pathway by the ubiquitin proteasome system is less explored. In this review we will focus on the recent findings about feedback regulation of ABA signaling core proteins through degradation, which is emerging as a critical step that modulates and eventually ceases the signal relay. Additionally, we also discuss the importance of the recently identified effector protein $\mathrm{HOS15}$, which negatively regulate ABA-signaling through degradation of OST1.

Keywords: ABA-signaling, ABA core proteins, protein degradation and stability, HOS15, OST1

\section{INTRODUCTION}

Being sessile by nature, plants have evolved the ability to alter their physiology and development to adapt to the environmental challenges (Bohnert et al., 1995). Unfavorable conditions, such as high salinity, cold or drought stress, are important challenges to agriculture as they reduce the yield potential of crop plants. Phytohormones play a pivotal role in environmental adaptation by inducing many biochemical and physiological changes to respond to biotic and abiotic stresses (Cao et al., 2011; Seo et al., 2014; Pozo et al., 2015). Among them, abscisic acid (ABA) is an important regulator of plant growth and development that also plays a crucial role in both biotic and abiotic stress responses (Lee et al., 2006; Adie et al., 2007; Mang et al., 2012; Finkelstein, 2013). ABA regulates multiple physiological processes such as seed maturation, embryo morphogenesis, and stomatal movement to rescue plants under water deficit condition (Yoshida et al., 2002; Finkelstein, 2013; Murata et al., 2015). The phenotypes of the ABA-defective mutants (both in synthesis and/or signaling), which included loss of seed dormancy and early seedling growth and loss of stomatal movement, supported the importance of ABA in developmental and physiological responses (Koornneef et al., 1982; Lee et al., 2006; Finkelstein, 2013). 
In response to environmental stresses $\mathrm{ABA}$ levels rise and set in motion the adaptive stress responses (Zabadal, 1974; Lee et al., 2006; Hua et al., 2012). The perception of ABA is achieved by a family of ABA receptors named Pyrabactin Resistance (PYR), or Regulatory Component of ABA Receptor (RCAR) (Ma et al., 2009; Park et al., 2009). In the presence of ABA, the ABA receptors PYR/PYL(PYR-Like)/RCAR function at the apex of a negative regulatory pathway to directly bind to and inactivate type 2C Ser/Thr protein phosphatases (PP2Cs) (Geiger et al., 2009; Ma et al., 2009; Park et al., 2009; Rodrigues et al., 2013). This allows the activation of SnRK2 kinases (Snf1-related protein kinase class 2), which subsequently phosphorylate ABI5/ABFs transcription factors (ABA-Insensitive5/ABA-responsive element binding factors) (Fujii et al., 2009). However, negative effectors that modulate the intensity of the response, and which will eventually cancel the signaling cascade, must also come into action to counteract this positive signal relay and prevent a runaway process. Finely controlled protein stability is emerging as a novel critical regulatory layer accounting for signal termination, and also for desensitization upon repeated or sustained stimuli. Here, we summarize recent insights into controlled protein degradation of signaling effectors of the ABA pathway, with an emphasis on the newly discovered player HOS15, a component of the protein ubiquitination machinery that tags SnRK2.6/OST1 and histone deacetylase 2C (HD2C) for degradation, thereby modulating ABA signaling, chromatin status and gene expression in response to dehydration and cold stresses in Arabidopsis (Park et al., 2018; Ali et al., 2019).

\section{UBIQUITIN PROTEASOME SYSTEM (UPS)}

Protein degradation by the Ubiquitin Proteasome System (UPS) is an important posttranslational regulatory step that controls protein stability and turnover. Protein ubiquitination combines activities of three enzymes, E1 (ubiquitin-activation), E2 (ubiquitin-conjugation), and E3 (ubiquitin ligase) (Pintard et al., 2004; Hotton and Callis, 2008). Target specificity is conferred by E3 ligases, and hence the Arabidopsis genome contains over 1400 genes encoding E3 ligases, which are classified into two groups (Vierstra, 2009). One group acts as a single subunit, which consists of RING-type (Really Interesting New Gene) E3 enzymes. The other group, which functions as a multi subunit complex, includes SCF (Skp1-Cullin-F-box) and APC (Anaphase Promoting Complex) (Vierstra, 2009). In the recent past, a number of RING-type E3 ligases were identified in Arabidopsis that were shown to be involved in various cellular processes, such as hormones signaling (auxin and ABA), seed germination and early seedling development and adaptive pathway to water limitation (Xie et al., 2002; Zhang et al., 2005, 2008; Stone et al., 2006; Bu et al., 2009; Huang et al., 2010). Among them, CULLIN4 (CUL4) has been described in greater detail due to its major role in different biological pathways. CUL4 interacts with DDB1 (DAMAGED DNA BINDING PROTEIN1) and a WD40-repeat protein as its substrate receptor (He et al., 2006; Hotton and Callis, 2008; Hua and Vierstra, 2011; Seo et al., 2014). The WD40-repeat (also known as the $B$-transducin repeat) is a structural motif that folds as a solenoid-like structure called the WD40 domain. WD40-repeat proteins function as to facilitate multi-protein complex assemblies, where they serve as the scaffold for protein-protein interactions, including those of E3 ubiquitin ligases with target proteins. The Arabidopsis protein HOS15 (HIGH OSMOTIC STRESS GENE EXPRESSION 15) is a substrate receptor for the CULLIN4 (CUL4)-based ubiquitin E3 ligase that plays a negative role on ABA signaling and plant acclimation to cold (Lee et al., 2008; Zhu et al., 2008; Park et al., 2018). In the cold-stress response, HOS15 functions to mediate the cold-induced degradation of histone deacetylase $2 \mathrm{C}$ (HD2C) in the promoters of the COLD-RESPONSIVE (COR) genes. Enhanced histone acetylation switches the chromatin from an "open" status that facilitates recruitment of CBF (C-REPEAT BINDING FACTOR) transcription factors to the COR genes (Park et al., 2018). We have recently shown that HOS15 also plays a substantial role in regulating the signaling flux in response to ABA by controlling the protein stability and abundance of intermediaries in the pathway (Ali et al., 2019).

PYR/PYL/RCAR (ABA receptors), PP2Cs (phosphatases), SnRKs (SNF1-related protein kinases) and ABI5/ABFs (transcription factors) are major components of forward ABA-signaling (Fujii and Zhu, 2009; Park et al., 2009). Ten SnRK2 members (SnRK2.1 to SnRK2.10) have been identified in Arabidopsis (Hrabak et al., 2003), with three of them (SnRK2.2/2.3/2.6) being activated by ABA (Fujita et al., 2009). Among the three, SnRK2.6/OST1 specifically regulates water loss through stomata (Yoshida et al., 2002; Hua et al., 2012). Evidence is emerging that ubiquitination and degradation of these ABA-signaling components is of upmost importance to fully understand that how this signaling pathway is modulated and eventually ceased (Table 1).

TABLE 1 | List of E3 ligases (and other proteins/linkers) which regulate protein level of ABA signaling core components.

\begin{tabular}{lll}
\hline E3 ligases/Linkers & Target protein & Published by \\
\hline AFP1 & ABI5 & Lopez-Molina et al., 2003 \\
DWA1/DWA2 & ABI5 & Lee et al., 2010 \\
KEG & ABI5/ABF1/3 & Liu and Stone, 2013; \\
& & Chen et al., 2013 \\
ABD1 & ABI5 & Seo et al., 2014 \\
RSL1 & PYL4/PYR1 & Bueso et al., 2014 \\
DDA1 & PYL4/8/9 & Irigoyen et al., 2014 \\
ALIX & PYL4/5/8/L9 & García-León et al., 2019 \\
RIFP1 & RCAR3 & Li et al., 2016 \\
REA1 & PYL9 & Li et al., 2018 \\
PUB22/PUB23 & PYL9 & Zhao et al., 2017 \\
FYV1 & PYL4 & Belda-Palazon et al., 2016 \\
VPS23A & PYR1/PYL4 & Yu et al., 2016 \\
PUB12/PUB13 & ABl1 & Kong et al., 2015 \\
RGLG1/RGLG5 & PP2CA & Wu et al., 2016 \\
PIR1/PIR2 & PP2CA & Baek et al., 2019 \\
BPM3/BPM5 & AB11/PP2CA/HAB1 & Julian et al., 2019 \\
PP2B11 & SnRK2.3 & Cheng et al., 2017 \\
HOS15 & OST1 & Ali et al., 2019
\end{tabular}




\section{ABA RECEPTORS (PYR1/PYL/RCAR)}

Several reports in the recent past have shown that $A B A$ receptors are degraded by a number of E3 ligases in an ABA-dependent manner (Table 1). Sorting and vacuolar degradation of ABA receptors are mediated by components of the ESCRT machinery (Endosomal Sorting Complex Required for Transport), i.e., FYVE1 and VPS23A (Belda-Palazon et al., 2016; Yu et al., 2016). ALIX (ALG-2 INTERACTING PROTEIN-X), another ESCRT protein, directly interact with ABA-receptors (PYL4/5/8/9) in late endosomes and promote their degradation (GarcíaLeón et al., 2019). Furthermore, genetic interference with ALIX function leads to altered endosomal localization and increased accumulation of $\mathrm{ABA}$ receptors, indicating that to perform normal function, inhibition of ABA-receptor's overaccumulation needs to be carried out (García-León et al., 2019). Degradation of RCAR1/PYL9 mediated by PUB22 and PUB23 (U-box E3 ligases) has recently been shown (Zhao et al., 2017). Additionally, RAE1, a WD40 repeat protein, and
RIFP1, an adaptor subunit of the SCF ubiquitin ligase complex, promote the degradation of RCAR1 and RCAR3, respectively (Li et al., 2016, 2018). Based on these reports one can easily assume that regardless of their positive role, controlled turnover of ABA-receptors at protein level is a critical step that fine tunes ABA-signaling pathway. The reason why degradation of the $\mathrm{ABA}$ receptors and of other signaling intermediaries (Table 1) is redundantly achieved by several types of E3 ligases is presently unknown. Likely, seemingly redundant ways leading to protein degradation reflect the diversity of developmental and physiological processes in which the target proteins participate or their diverse subcellular locations (see below).

\section{PROTEIN PHOSPHATASES (ABI/HAI/HAB)}

Next to ABA receptors, protein phosphatases (PP2Cs), acting as co-receptors of $\mathrm{ABA}$, have emerged as important regulators

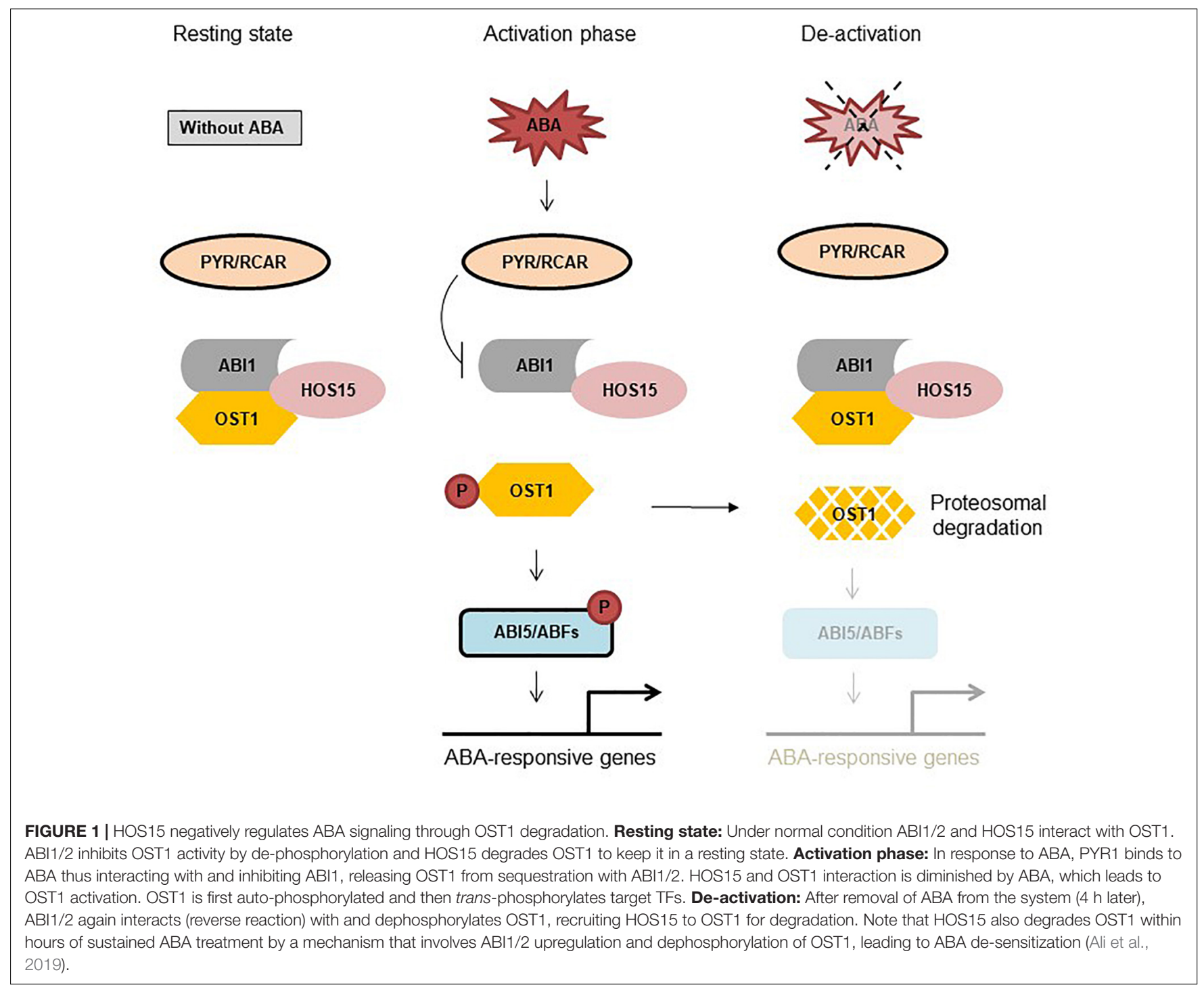


of ABA-signaling. Primarily, PP2Cs function as inhibitors of ABA-signaling pathway through inactivation of SnRK2 kinases (Brandt et al., 2012). Members of protein phosphatases PP2Cs that include $\mathrm{ABI} 1 / 2, \mathrm{HAB} 1 / 2, \mathrm{HAI} 1 / 2 / 3$, and $\mathrm{AHG} 1 / 3$ sequester SnKR2 kinases, inhibiting their kinase activity and thus functions as negative regulators of $\mathrm{ABA}$-signaling. To release from sequestration by PP2Cs, SnRK2 kinases require ABA binding to PYR1/PYL/RCAR receptors (Fujii et al., 2009). Furthermore, increased or decreased phosphatase activities of these PP2Cs result in altered ABA responses that are SnRK2-dependent. For instance, knock out mutants of PP2Cs such as abi1-2 display enhanced ABA signaling, whereas dominant-negative mutations of the same PP2C (abi1-1), lead to suppression of ABA-signaling in a SnRK2s-dependent manner (Umezawa et al., 2009). PP2Cs are also regulated through proteasomal degradation. Degradation of ABI1, a well-known protein phosphatase in the ABA-signaling, has been reported (Kong et al., 2015). PUB12/PUB13 (U-box E3 ligases) interact with $\mathrm{ABI} 1$ and are able to ubiquitinate $\mathrm{ABI} 1$ in the presence of ABA. The ubiquitinated ABI1 is degraded by the $26 \mathrm{~S}$ proteasome (Kong et al., 2015). Beside ABI1, the degradation of PP2CA, another phosphatase that negatively regulate SnRK2s activity, was recently reported, which is mediated by the RING finger E3 ligases RGLG1/RGLG5 and PIR1/PIR2 (Wu et al., 2016; Baek et al., 2019). In all these cases, E3 ligases positively modulate ABA signaling by targeting the phosphatases for degradation, thereby amplifying the signal flux.

As with the ABA receptors, more than one E3 ligase modifies a single substrate depending on the physiological context (Hare et al., 2003; Liu and Stone, 2010; Cheng et al., 2012; Seo et al., 2014). For instance, PP2CA protein stability is modulated by both RGLG1/5 and PIR1/2 (Wu et al., 2016; Baek et al., 2019). However, compared with RGLG1/5, PIR1 and PIR2 can strongly interact with PP2CA in the absence or the presence of low ABA, suggesting that PIR1 and PIR2 may regulate PP2CA protein stability under non-stress conditions (Wu et al., 2016; Baek et al., 2019).

\section{Snf1-RELATED KINASES2 (SnRK2s)}

Although Snf1-Related Kinases (SnRK2s) kinases are major components that regulate ABA-signaling pathway, little is known about the feedback regulation of SnRK2s in post-ABA condition to terminate the signal. Overall, de-activation of SnRK2s at protein level, has been less studied and only few reports are available to date (Table 1). Upon ABA signal, activated SnRK2s phosphorylate and activate target proteins including $\mathrm{ABF}$ transcription factors (Fujita et al., 2009). Interestingly, the activated ABFs bind to the promoters of $A B I 1$ and $A B I 2$ genes and promote their transcription, thereby controlling SnRK2s activity through a negative feedback regulation loop (Wang et al., 2018). Besides dephosphorylation by PP2Cs, AtPP2-B11, a component of the SCF ubiquitin E3 ligase complex, has been shown to promote the ABA-dependent ubiquitination and degradation of SnRK2.3 (Cheng et al., 2017). More recently, we have also shown that the degradation of SnRK2 kinases is really important for the controlled turnover of ABA signal relay. Using yeast two hybrid screen, we identified that HOS15 interacts specifically with OST1, SnRK2.3 and ABI1/2 (and very weakly with HAI1). Further analysis showed that HOS15 specifically interacts with OST1 in an ABA-dependent manner and promotes its degradation (Ali et al., 2019). OST1 level was highly accumulated in hos 15-2 plants, which leads to significant tolerance to drought stress (Ali et al., 2019).

\section{INVOLVEMENT OF HOS15 IN ABA-SIGNALING PATHWAY}

HOS15, a substrate receptor in the CUL4-DDB1 E3 ligase machinery, negatively regulate $\mathrm{ABA}$-signaling and drought stress by interference with OST1 stability (Ali et al., 2019). HOS15 was found to interact with ABI1, ABI2, and OST1; however, only HOS15 and OST1 interaction was diminished by ABA (Ali et al., 2019). OST1 level was remarkably stable and accumulated in hos 15-2 compared to wild type (Columbia-0) plants, demonstrating that HOS15 negatively regulates OST1 stability, presumably leading to termination of ABA signaling (Ali et al., 2019). Loss-of-function hos15-2 mutant plants are hypersensitive to ABA during germination and extremely tolerant to

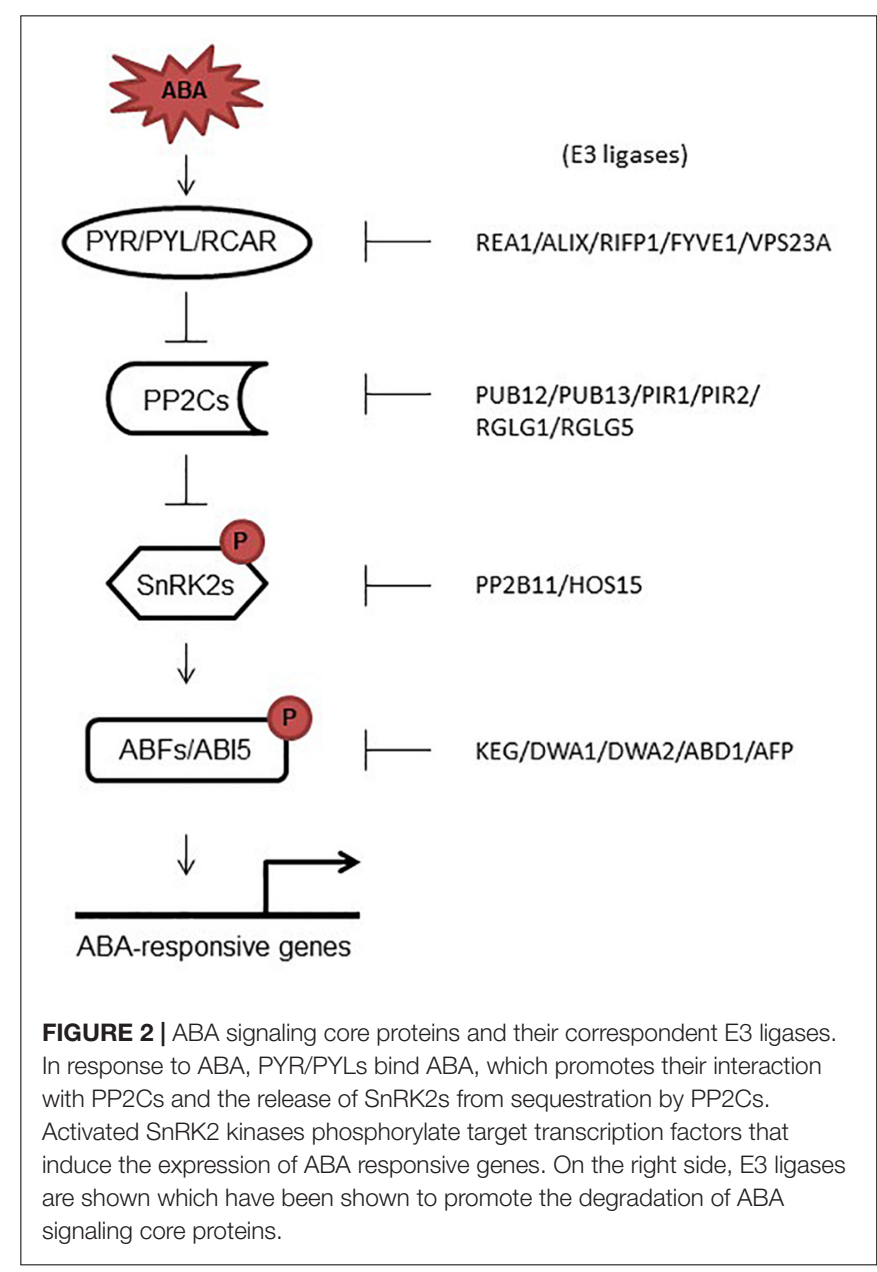


drought stress, indicating the importance of HOS15 as a negative regulator (Ali et al., 2019). Moreover, ABA- and dehydration stress-responsive genes were highly induced in hos 15-2 plants under dehydration stress (Ali et al., 2019). Since HOS15 plays a major role in ABA-signaling network, we were interested to place HOS15 in the current model of ABA-signaling pathway. Under normal condition ABI1/2 inhibits OST1 activity by dephosphorylating it (Yoshida et al., 2006; Park et al., 2009). In the presence of ABA, PYR1 inhibits ABI1, releasing OST1 that auto-phosphorylates itself and then trans-phosphorylate the target TFs (Antoni et al., 2012). Furthermore, ABA also impairs HOS15 and OST1 interaction (Ali et al., 2019), indicating that in the presence of ABA, inhibitory components are kept stay-away from OST1 (and other SnRK2s) that phosphorylate and activates $\mathrm{ABA}$ responsive components. By contrast, $\mathrm{ABA}$ has no clear effect on HOS15 interaction with ABI1 and ABI2 (Ali et al., 2019), demonstrating that once the ABA pathway is activated, OST1 is released from the HOS15-ABI1/2 complex. However, when ABA-pathway is about to turn-off, ABI1/2 promotes HOS 15 and OST1 interaction, which leads to OST1 degradation (Figure 1). Importantly, we found that under sustained ABA stimulus, activated OST1 promoted de novo synthesis and accumulation of $\mathrm{ABI} 1 / 2$, which in turn dephosphorylated and promoted the degradation of OST1 (Figure 1). Accordingly, de-phosphorylated OST1 was the preferred substrate for HOS15 (Ali et al., 2019). Together, these functional and physical interactions depict the activity of a biological rheostat that through quantitative and mutual regulation of both positive and negative effectors achieves the adaptive modulation of signal amplitude and duration. In summary, HOS15 plays a crucial role in regulating ABA-signaling by degradation of OST1 and thus keeping a balance between active and inactive state.

\section{ABFs/ABI5}

Activation of ABFs/ABI5 transcription factors (TFs) by SnRK2s completes the signal relay and links ABA signaling with ABA-dependent gene activation. Like their activation by phosphorylation, degradation of ABFs/ABI5 TFs by $26 \mathrm{~S}$ proteasome has also been studied in detail (Table $\mathbf{1}$ and Figure 2). The first evidence regarding degradation of ABFs/ABI5 TFs was the identification of ABI FIVE BINDING PROTEIN1 (AFP1), a member of a small plant-specific protein family. AFP1 directly binds to ABI5 and facilitates ubiquitin-mediated proteolysis of ABI5 (Lopez-Molina et al., 2003). More recently, a number

\section{REFERENCES}

Adie, B. A., Pérez-Pérez, J., Pérez-Pérez, M. M., Godoy, M., Sánchez-Serrano, J. J., Schmelz, E. A., et al. (2007). ABA is an essential signal for plant resistance to pathogens affecting JA biosynthesis and the activation of defenses in Arabidopsis. Plant Cell 19, 1665-1681. doi: 10.1105/tpc.106.048041

Ali, A., Kim, J., Jan, M., Khan, H. A., Khan, I. U., Shen, M., et al. (2019). Rheostatic control of ABA signaling through HOS15-mediated OST1 degradation. Mol. Plant 12, 1447-1462. doi: 10.1016/j.molp.2019.08.005 of E3 ligases and WD40-repeat proteins have been identified as negative regulators of both ABFs and ABI5 (Table 1). KEG (KEEP ON GOING), a RING-ANK E3 ligase, is required for the regulation of $\mathrm{ABI} 5 / \mathrm{ABF} 1 / \mathrm{ABF} 3$ abundance (Chen et al., 2013; Liu and Stone, 2013). In vivo studies have shown that in the absence of $\mathrm{ABA}$, KEG ubiquitinate $\mathrm{ABF} 1 / \mathrm{ABF} 3 / \mathrm{ABI} 5$, and promotes proteasomal degradation of them (Liu and Stone, 2013). In addition, DWA1 and DWA2 (DWD hypersensitive to ABA1/2) are substrate receptors for the DDBI CULLIN4-based E3 ligases that directly interact with ABI5 and mediate the degradation of ABI5 by ubiquitination (Lee et al., 2010). More recently, Seo et al. (2014) found that ABD1, a WD40-repeat protein, directly interacts with and degrades $\mathrm{ABI} 5$. These reports indicate that activation and degradation of ABA-signaling components are critical processes that regulate ABA signaling pathway in a very fine way and that both processes are important alike.

\section{CONCLUSION}

The plant hormone abscisic acid (ABA) controls a number of developmental processes including seed maturation, germination, embryogenesis, plant growth and development, and senescence (Zabadal, 1974; Lee et al., 2006; Finkelstein, 2013; Murata et al., 2015; Zhao et al., 2016). Several proteins were identified that work together to regulate ABA signaling including ABA-receptors and co-receptors, kinases and TFs (Fujii et al., 2009; Fujita et al., 2009; Park et al., 2009; Brandt et al., 2012). Activation and de-activation (degradation) of these proteins got huge attention in the recent past (Figure 2); however, compared to activation, little is known about degradation of these proteins. This review sheds light on the recent studies that focused on the degradation of ABA-signaling core components. Further investigations on how different E3 ligases are activated to degrade ABA core proteins, are the future goals.

\section{AUTHOR CONTRIBUTIONS}

D-JY and JP designed work. AA, JP, and D-JY collected information from literature and wrote the manuscript. All authors reviewed and approved the final manuscript.

\section{FUNDING}

This work was supported by the Konkuk University in 2018. 
receptor and mediates its delivery to the vacuolar degradation pathway. Plant Cell 28, 2291-2311. doi: 10.1105/tpc.16.00178

Bohnert, H. J., Nelson, D. E., and Jensen, R. G. (1995). Adaptations to environmental stresses. Plant Cell 7, 1099-1111. doi: 10.1105/tpc.7.7.1099

Brandt, B., Brodsky, D. E., Xue, S., Negi, J., Iba, K., Kangasjärvi, J., et al. (2012). Reconstitution of abscisic acid activation of SLAC1 anion channel by CPK6 and OST1 kinases and branched ABI1 PP2C phosphatase action. Proc. Natl. Acad. Sci. U.S.A. 109, 10593-10598. doi: 10.1073/pnas.1116590109

Bu, Q., Li, H., Zhao, Q., Jiang, H., Zhai, Q., Zhang, J., et al. (2009). The Arabidopsis RING finger E3 ligase RHA2a is a novel positive regulator of abscisic acid signaling during seed germination and early seedling development. Plant Physiol. 150, 463-481. doi: 10.1104/pp.109.135269

Bueso, E., Rodriguez, L., Lorenzo-Orts, L., Gonzalez-Guzman, M., Sayas, E., Munoz-Bertomeu, J., et al. (2014). The single-subunit RING-type E3 ubiquitin ligase RSL1 targets PYL4 and PYR1 ABA receptors in plasma membrane to modulate abscisic acid signaling. Plant J. 80, 1057-1071. doi: 10.1111/tpj.12708

Cao, F. Y., Yoshioka, K., and Desveaux, D. (2011). The roles of ABA in plantpathogen interactions. J. Plant Res. 124, 489-499. doi: 10.1007/s10265-0110409-y

Chen, Y. T., Liu, H., Stone, S., and Callis, J. (2013). ABA and the ubiquitin E3 ligase KEEP ON GOING affect proteolysis of the Arabidopsis thaliana transcription factors ABF1 and ABF3. Plant J. 75, 965-976. doi: 10.1111/tpj.12259

Cheng, C., Wang, Z., Ren, Z., Zhi, L., Yao, B., Su, C., et al. (2017). SCFAtPP2-B11 modulates ABA signaling by facilitating SnRK2.3 degradation in Arabidopsis thaliana. PLoS Genet. 13:e1006947. doi: 10.1371/journal.pgen.1006947

Cheng, M. C., Hsieh, E. J., Chen, J. H., Chen, H. Y., and Lin, T. P. (2012). Arabidopsis RGLG2, functioning as a RING E3 ligase, interacts with AtERF53 and negatively regulates the plant drought stress response. Plant Physiol. 158, 363-375. doi: 10.1104/pp.111.189738

Finkelstein, R. (2013). Abscisic acid synthesis and response. Arabidopsis Book 11:e0166. doi: 10.1199/tab.0166

Fujii, H., Chinnusamy, V., Rodrigues, A., Rubio, S., Antoni, R., and Park, S. Y. (2009). In vitro reconstitution of an abscisic acid signalling pathway. Nature. 3:462. doi: $10.1038 /$ nature 08599

Fujii, H., and Zhu, J. K. (2009). Arabidopsis mutant deficient in 3 abscisic acid-activated protein kinases reveals critical roles in growth, reproduction, and stress. Proc. Natl Acad. Sci. U.S.A. 106, 8380-8385. doi: 10.1073/pnas. 0903144106

Fujita, Y., Nakashima, K., Yoshida, T., Katagiri, T., Kidokoro, S., Kanamori, N., et al. (2009). Three SnRK2 protein kinases are the main positive regulators of abscisic acid signaling in response to water stress in Arabidopsis. Plant Cell Physiol. 50, 2123-2132. doi: 10.1093/pcp/pcp147

García-León, M., Cuyas, L., El-Moneim, D. A., Rodriguez, L., Belda-Palazón, B., Sanchez-Quant, E., et al. (2019). Arabidopsis ALIX regulates stomatal aperture and turnover of abscisic acid receptors. Plant Cell 31, 2411-2429. doi: 10.1105/ tpc. 19.00399

Geiger, D., Scherzer, S., Mumm, P., Stange, A., Marten, I., Bauer, H., et al. (2009). Activity of guard cell anion channel SLAC1 is controlled by drought-stress signaling kinase-phosphatase pair. Proc. Natl Acad. Sci. U.S.A. 106, 2142521430. doi: $10.1073 /$ pnas.0912021106

Hare, P. D., Seo, H. S., Yang, J. Y., and Chua, N. H. (2003). Modulation of sensitivity and selectivity in plant signaling by proteasomal destabilization. Curr. Opin. Plant Biol. 6, 453-462. doi: 10.1016/s1369-5266(03)00080-3

He, Y. J., McCall, C. M., Hu, J., Zeng, Y., and Xiong, Y. (2006). DDB1 functions as a linker to recruit receptor WD40 proteins to CUL4-ROC1 ubiquitin ligases. Genes Dev. 20, 2949-2954. doi: 10.1101/gad.1483206

Hotton, S. K., and Callis, J. (2008). Regulation of cullin RING ligases. Annu. Rev. Plant Biol. 59, 467-489. doi: 10.1146/annurev.arplant.58.032806.104011

Hrabak, E. M., Chan, C. W., Gribskov, M., Harper, J. F., Choi, J. H., Halford, N., et al. (2003). The Arabidopsis CDPK-SnRK superfamily of protein kinases. Plant Physiol. 132, 666-680. doi: 10.1104/pp.102.011999

Hua, D., Wang, C., He, J., Liao, H., Duan, Y., Zhu, Z., et al. (2012). A plasma membrane receptor kinase, GHR1, mediates abscisic acid- and hydrogen peroxide-regulated stomatal movement in Arabidopsis. Plant Cell 24, 25462561. doi: $10.1105 /$ tpc.112.100107

Hua, Z., and Vierstra, R. D. (2011). The cullin-RING ubiquitin-protein ligases. Annu. Rev. Plant Biol. 62, 299-334. doi: 10.1146/annurev-arplant-042809112256
Huang, Y., Li, C. Y., Pattison, D. L., Gray, W. M., Park, S., and Gibson, S. I. (2010). SUGARINSENSITIVE3, a RING E3 ligase, is a new player in plant sugar response. Plant Physiol. 152, 1889-1900. doi: 10.1104/pp.109.150573

Irigoyen, M. L., Iniesto, E., Rodriguez, L., Puga, M. I., Yanagawa, Y., Pick, E., et al. (2014). Targeted degradation of abscisic acid receptors is mediated by the ubiquitin ligase substrate adaptor DDA1 in Arabidopsis. Plant Cell 26, 712-728. doi: $10.1105 /$ tpc. 113.122234

Julian, J., Coego, A., Lozano Juste, J., Lechner, E., Wu, Q., Zhang, X., et al. (2019). The MATH-BTB BPM3 and BPM5 subunits of cullin3-RING E3 ubiquitin ligases target PP2CA and other clade A PP2Cs for degradation. Proc. Natl. Acad. Sci. U.S.A. 116, 15725-15734. doi: 10.1073/pnas.1908677116

Kong, L., Cheng, J., Zhu, Y., Ding, Y., Meng, J., Chen, Z., et al. (2015). Degradation of the ABA co-receptor ABI1 by PUB12/13 U-box E3 ligases. Nat. Commun. 6:8630. doi: $10.1038 /$ ncomms 9630

Koornneef, M., Jorna, M. L., Brinkhorst van der Swan, D. L., and Karssen, C. M. (1982). The isolation of abscisic acid (ABA) deficient mutants by selection of induced revertants in nongerminating gibberellin sensitive lines of Arabidopsis thaliana (L.) heynh. Theor. Appl. Genet. 61, 385-393. doi: 10.1007/bf00272861

Lee, J. H., Terzaghi, W., Gusmaroli, G., Charron, J. B., Yoon, H. J., Chen, H., et al. (2008). Characterization of Arabidopsis and rice DWD proteins and their roles as substrate receptors for CUL4-RING E3 ubiquitin ligases. Plant Cell 20, 152-167. doi: 10.1105/tpc.107.055418

Lee, J. H., Yoon, H. J., Terzaghi, W., Martinez, C., Dai, M., Li, J., et al. (2010) DWA1 and DWA2, two Arabidopsis DWD protein components of CUL4-based E3 ligases, act together as negative regulators in ABA signal transduction. Plant Cell 22, 1716-1732. doi: 10.1105/tpc.109.073783

Lee, K. H., Piao, H. L., Kim, H.-Y., Choi, S. M., Jiang, F., Hartung, W., et al. (2006). Activation of glucosidase via stress-induced polymerization rapidly increases active pools of abscisic acid. Cell 126, 1109-1120. doi: 10.1016/j.cell.2006.07.034

Li, D., Zhang, L., Li, X., Kong, X., Wang, X., Li, Y., et al. (2018). AtRAE1 is involved in degradation of $\mathrm{ABA}$ receptor $\mathrm{RCAR} 1$ and negatively regulates $\mathrm{ABA}$ signalling in Arabidopsis. Plant Cell Environ. 41, 231-244. doi: 10.1111/pce.13086

Li, Y., Zhang, L., Li, D., Liu, Z., Wang, J., Li, X., et al. (2016). The Arabidopsis F-box E3 ligase RIFP1 plays a negative role in abscisic acid signalling by facilitating ABA receptor RCAR3 degradation. Plant Cell Environ. 39, 571-582. doi: $10.1111 /$ pce. 12639

Liu, H., and Stone, S. L. (2010). Abscisic acid increases Arabidopsis ABI5 transcription factor levels by promoting KEG E3 ligase self-ubiquitination and proteasomal degradation. Plant Cell 22, 2630-2641. doi: 10.1105/tpc.110. 076075

Liu, H., and Stone, S. L. (2013). Cytoplasmic degradation of the Arabidopsis transcription factor abscisic acid insensitive 5 is mediated by the RING-type E3 ligase KEEP ON GOING. J. Biol. Chem. 288, 20267-20279. doi: 10.1074/jbc. M113.465369

Lopez-Molina, L., Mongrand, S., Kinoshita, N., and Chua, N. H. (2003). AFP is a novel negative regulator of ABA signaling that promotes ABI5 protein degradation. Genes Dev. 17, 410-418. doi: 10.1101/gad.1055803

Ma, Y., Szostkiewicz, I., Korte, A., Moes, D., Yang, Y., Christmann, A., et al. (2009). Regulators of PP2C phosphatase activity function as abscisic acid sensors. Science 324, 1064-1068. doi: 10.1126/science.1172408

Mang, H. G., Qian, W., Zhu, Y., Qian, J., Kang, H. G., Klessig, D. F., et al. (2012). Abscisic acid deficiency antagonizes high-temperature inhibition of disease resistance through enhancing nuclear accumulation of resistance proteins SNC1 and RPS4 in Arabidopsis. Plant Cell 24, 1271-1284. doi: 10.1105/tpc.112. 096198

Murata, Y., Mori, I. C., and Munemasa, S. (2015). Diverse stomatal signaling and the signal integration mechanism. Annu. Rev. Plant Biol. 66, 369-392. doi: 10.1146/annurev-arplant-043014-114707

Park, J., Lim, C. J., Shen, M., Park, H. J., Cha, J. Y., Iniesto, E., et al. (2018) Epigenetic switch from repressive to permissive chromatin in response to cold stress. Proc. Natl. Acad. Sci. U.S.A. 115, 5400-5409. doi: 10.1073/pnas. 1721241115

Park, S. Y., Fung, P., Nishimura, N., Jensen, D. R., Fujii, H., Zhao, Y., et al. (2009). Abscisic acid inhibits type $2 \mathrm{C}$ protein phosphatases via the PYR/PYL family of START proteins. Science 324, 1068-1071. doi: 10.1126/science.1173041

Pintard, L., Willems, A., and Peter, M. (2004). Cullin-based ubiquitin ligases: cul3BTB complexes join the family. EMBO J. 23, 1681-1687. doi: 10.1038/sj.emboj. 7600186 
Pozo, M. J., López-Ráez, J. A., Azcón, C., and García-Garrido, J. M. (2015). Phytohormones as integrators of environmental signals in the regulation of mycorrhizal symbioses. New Phytol. 205, 1431-1436. doi: 10.1111/nph.13252

Rodrigues, A., Adamo, M., Crozet, P., Margalha, L., Confraria, A., Martinho, C., et al. (2013). ABI1 and PP2CA phosphatases are negative regulators of snf1related protein kinasel signaling in Arabidopsis. Plant Cell 25, 3871-3884. doi: 10.1105/tpc.113.114066

Seo, K. I., Lee, J. H., Nezames, C. D., Zhong, S., Song, E., Byun, M. O., et al. (2014). ABD1 is an Arabidopsis DCAF substrate receptor for CUL4-DDB1-based E3 ligases that acts as a negative regulator of abscisic acid signaling. Plant Cell 26, 695-711. doi: 10.1105/tpc.113.119974

Stone, S. L., Williams, L. A., Farmer, L. M., Vierstra, R. D., and Callis, J. (2006). KEEP ON GOING, a RING E3 ligase essential for Arabidopsis growth and development, is involved in abscisic acid signaling. Plant Cell 18, 3415-3428. doi: 10.1105/tpc.106.046532

Umezawa, T., Sugiyama, N., Mizoguchi, M., Hayashi, S., Myouga, F., YamaguchiShinozaki, K., et al. (2009). Type 2C protein phosphatases directly regulate abscisic acid-activated protein kinases in Arabidopsis. Proc. Natl. Acad. Sci. U.S.A. 106, 17588-17593. doi: 10.1073/pnas.0907095106

Vierstra, R. D. (2009). The ubiquitin-26S proteasome system at the nexus of plant biology. Nat. Rev. Mol. Cell Biol. 10, 385-397. doi: 10.1038/nrm2688

Wang, X., Guo, C., Peng, J., Li, C., Wan, F., Zhang, S., et al. (2018). ABREBINDING FACTORS play a role in the feedback regulation of ABA signaling by mediating rapid ABA induction of ABA co-receptor genes. New Phytol. 221, 341-355. doi: 10.1111/nph.15345

Wu, Q., Zhang, X., Peirats-Llobet, M., Belda-Palazon, B., Wang, X., Cui, S., et al. (2016). Ubiquitin ligases RGLG1 and RGLG5 regulate abscisic acid signaling by controlling the turnover of phosphatase PP2CA. Plant Cell 28, 2178-2196. doi: $10.1105 /$ tpc. 16.00364

Xie, Q., Guo, H. S., Dallman, G., Fang, S., Weissman, A. M., and Chua, N. H. (2002). SINAT5 promotes ubiquitin-related degradation of NAC1 to attenuate auxin signals. Nature 419, 167-170. doi: 10.1038/nature00998

Yoshida, R., Hobo, T., Ichimura, K., Mizoguchi, T., Takahashi, F., Aronso, J., et al. (2002). ABA-activated SnRK2 protein kinase is required for dehydration stress signaling in Arabidopsis. Plant Cell Physiol. 43, 1473-1483. doi: 10.1093/pcp/ pcf188

Yoshida, R., Umezawa, T., Mizoguchi, T., Takahashi, S., Takahashi, F., and Shinozaki, K. (2006). The regulatory domain of SRK2E/OST1/ SnRK2.6 interacts with $A B I 1$ and integrates abscisic acid (ABA) and osmotic stress signals controlling stomatal closure in Arabidopsis. J. Biol. Chem. 281, 5310-5318. doi: 10.1074/jbc.m509820200

Yu, F., Lou, L., Tian, M., Li, Q., Ding, Y., Cao, X., et al. (2016). ESCRT-I component VPS23A affects ABA signaling by recognizing ABA receptors for endosomal degradation. Mol. Plant. 9, 1570-1582. doi: 10.1016/j.molp.2016. 11.002

Zabadal, T. J. (1974). A water potential threshold for the increase of abscisic acid in leaves. Plant Physiol. 53, 125-127. doi: 10.1104/pp.53.1.125

Zhang, X., Garreton, V., and Chua, N. H. (2005). The AIP2 E3 ligase acts as a novel negative regulator of $\mathrm{ABA}$ signaling by promoting $\mathrm{ABI} 3$ degradation. Genes Dev. 19, 1532-1543. doi: 10.1101/gad.1318705

Zhang, Y., Xu, W., Li, Z., Deng, X. W., Wu, W., and Xue, Y. (2008). F-box protein DOR functions as a novel inhibitory factor for abscisic acid-induced stomatal closure under drought stress in Arabidopsis. Plant Physiol. 148, 2121-2133. doi: $10.1104 /$ pp.108.126912

Zhao, J., Zhao, L., Zhang, M., Zafar, S. A., Fang, J., Li, M., et al. (2017). Arabidopsis E3 ubiquitin ligases PUB22 and PUB23 negatively regulate drought tolerance by targeting ABA receptor PYL9 for degradation. Int. J. Mol. Sci. 18:E1841. doi: 10.3390/ijms18091841

Zhao, Y., Chan, Z., Gao, J., Xing, L., Cao, M., and Yu, C. (2016). ABA receptor PYL9 promotes drought resistance and leaf senescence. Proc. Natl. Acad. Sci. U.S.A. 113, 1949-1954. doi: 10.1073/pnas.1522840113

Zhu, J., Jeong, J. C., Zhu, Y., Sokolchik, I., Miyazaki, S., Zhu, J. K., et al. (2008). Involvement of Arabidopsis HOS15 in histone deacetylation and cold tolerance. Proc. Natl. Acad. Sci. U.S.A 105, 4945-4950. doi: 10.1073/pnas.0801029105 doi: $10.1073 /$ pnas.0801029105

Conflict of Interest: The authors declare that the research was conducted in the absence of any commercial or financial relationships that could be construed as a potential conflict of interest.

Copyright (C) 2020 Ali, Pardo and Yun. This is an open-access article distributed under the terms of the Creative Commons Attribution License (CC BY). The use, distribution or reproduction in other forums is permitted, provided the original author(s) and the copyright owner(s) are credited and that the original publication in this journal is cited, in accordance with accepted academic practice. No use, distribution or reproduction is permitted which does not comply with these terms. 